\title{
STOCHASTIC FLOWS WITH INTERACTION AND MEASURE-VALUED PROCESSES
}

\author{
ANDREY A. DOROGOVTSEV
}

Received 5 January 2003

\begin{abstract}
We consider the new class of the Markov measure-valued stochastic processes with constant mass. We give the construction of such processes with the family of the probabilities which describe the motion of single particles. We also consider examples related to stochastic flows with the interactions and the local times for such processes.
\end{abstract}

2000 Mathematics Subject Classification: 60H15, 60H10.

1. Introduction. This paper is devoted to one type of the Markov measurevalued processes with constant mass. We consider the measure-valued processes $\left\{\mu_{t} ; t \geq 0\right\}$ which have the following representation:

$$
\mu_{t}=\mu \circ f_{t}^{-1} \text {. }
$$

Here the random mapping $f_{t}$ on the state space depends on the initial state $\mu_{0}$. In the representation (1.1), $f_{t}$ can describe the motion on the phase space which carries the mass $\mu_{t}$. In such a model, the behavior of the trajectory which starts from a certain point depends on the whole mass distribution on the space. These processes are different from the well-known Fleming-Viot processes with constant mass. Consider, for example, the equation for the evolution of the discrete system of the particles, which was proposed in [3].

EXAMPLE 1.1. Let $a_{1}, \ldots, a_{N}$ be positive numbers and let $\{w(t) ; t \geq 0\}$ be a Wiener process in $\mathbb{R}^{d}$. Consider the following system of stochastic differential equations:

$$
\begin{gathered}
d x_{i}(t)=\sum_{j=1}^{N} a_{j} h\left(x_{i}(t), x_{j}(t)\right) d t+\varphi\left(x_{i}(t)\right) d t+b\left(x_{i}(t)\right) d w(t), \\
x_{i}(0)=x_{i}^{0}, \quad i=1, \ldots, N .
\end{gathered}
$$

Note that the first summand in the drift term can be rewritten in the form

$$
\sum_{j=1}^{N} a_{j} h\left(x_{i}(t), x_{j}(t)\right)=\int_{\mathbb{R}}^{d} h\left(x_{i}(t), u\right) \chi_{N, t}(d u),
$$


where $\chi_{N, t}$ is the discrete measure

$$
\chi_{N, t}=\sum_{j=1}^{N} a_{j} \delta_{x_{j}(t)} .
$$

It is well known that under appropriate conditions on the coefficients, system (1.2) has a unique strong solution for arbitrary initial conditions. It follows that the related measure-valued process $\left\{\chi_{N, t} ; t \geq 0\right\}$ is a Markov process. Moreover, in this case, we can find the function $f_{t}$ from (1.1). Consider the following stochastic differential equation together with (1.2):

$$
\begin{gathered}
d x(u, t)=\int_{\mathbb{R}} d h(x(u, t), v) \chi_{N, t}(d v) d t+\varphi(x(u, t)) d t+b(x(u, t)) d w(t), \\
x(u, 0)=u, \quad u \in \mathbb{R}^{d} .
\end{gathered}
$$

It can be checked that under Lipschitz and growth conditions on the coefficients, (1.5) has a unique solution which can be chosen continuously with respect to both variables with probability one. Now, note that

$$
x_{i}(t)=x\left(x_{i}^{0}, t\right)(\operatorname{modP}), \quad i=1, \ldots, N
$$

So,

$$
\chi_{N, t}=\chi_{N, 0} \circ x(\cdot, t)^{-1}
$$

In this example the random function $x$ describes the motion of the particles in the whole $\mathbb{R}^{d}$, but only $N$ of them have positive mass.

So, only certain values of $x$ are important if we consider the evolution of the mass distribution. The situation changes if the initial mass distribution is not the discrete measure. Also, in this example, the trajectory of the single particle is the Itô process, which satisfies certain stochastic differential equation. The particles which were disjoint in the time zero remain disjoint all the time.

EXAMPLE 1.2. Let the phase space $\mathfrak{X}=\{1, \ldots, N\}$. Consider the discrete time $t \in \mathbb{Z}_{+}$and define the motion of the system of particles on $\mathfrak{X}$ in the following way.

For every $i=1, \ldots, N$, let $\Pi(i)=\left(p_{k j}(i)\right)_{k j=1}^{N}$ be a stochastic matrix. Suppose that at time the mass $\mu_{t}=\left(\mu_{t}^{1}, \ldots, \mu_{t}^{N}\right)$ is distributed in $\mathfrak{X}$. Then, on the next step, all the particles which are placed at the point $k$ move together, independently from the past of all the system and from the particles placed at other points, to the point $j$ with probability

$$
\int_{\mathcal{X}} p_{k j}(i) \mu_{t}(d i)=\sum_{i=1}^{N} p_{k j}(i) \mu_{t}^{i} .
$$


Now we construct the process $\left\{\mu_{t} ; t \geq 0\right\}$ and the random mappings $\left\{f_{t} ; t \geq\right.$ $0\}$ in the following way. For every $i \in\{1, \ldots, N\}$, define $f_{t}(i)$ as a position which has the particle initially placed in $i$ at time $t$. Also define $\mu_{t}$ as a mass distribution at time $t$. It can easily be checked that now $\left\{\mu_{t} ; t \geq 0\right\}$ is a Markov process and, for every $t \geq 0$,

$$
\mu_{t}=\mu \circ f_{t}^{-1}
$$

Note that in this example the different particles may couple with one another and move together after coupling.

The aim of this paper is to define precisely the class of Markov measurevalued processes, which has the representation (1.1). Also, we consider a new type of stochastic differential equations which generalize Example 1.1. Correspondingly to this aim, the paper is organized as follows. In Section 2, we construct the measure-valued process with the desired property from the set of probabilities which describe the behavior of a finite number of particles. Section 3 contains the solution of stochastic differential equations driven by the measures and other examples of processes from Section 2. This contains the description of the local times for our processes.

2. Evolutionary measure-valued processes. Let $(\mathfrak{X}, \rho)$ be a complete separable metric space such that $\sup _{u, v \in \mathcal{X}} \rho(u, v)=1$, and let $(\Omega, \mathscr{F}, \mathrm{P})$ be a probability space. Denote by $\mathfrak{X}^{n}$ the $n$-tuple product $\mathfrak{X} \times \cdots \times \mathfrak{X}$ with the metrics

$$
\rho_{n}\left(\left(u_{1}, \ldots, u_{n}\right),\left(v_{1}, \ldots, v_{n}\right)\right)=\max _{i=1, \ldots, n} \rho\left(u_{i}, v_{i}\right)
$$

Let $\mathscr{B}_{n}$ be a Borel $\sigma$-field in $\mathfrak{*}^{n}$. Denote by $2 \mathfrak{n}_{n}$ the set of all probability measures on $\mathscr{B}_{n}$ (in the case $n=1$ the index will be omitted). For every $n \geq 1$, define the Wasserstein metrics in $\mathfrak{W}_{n}$ :

$$
\gamma_{n}(\mu, v)=\inf _{Q \in C(\mu, v)}\left(\iint_{\mathfrak{X}^{2 n}} \rho_{n}(x, y) Q(d x, d y)\right) \quad \forall \mu, \nu \in \mathfrak{W}_{n},
$$

where the infimum is taken over the set of all measures $C(\mu, v)$ which has $\mu$ and $v$ as its marginals. It is known that $\left(2 \mathfrak{V}_{n}, \gamma_{n}\right)$ is a complete separable metric space.

Let $\left\{\mathscr{F}_{t} ; t \geq 0\right\}$ be a flow of $\sigma$-fields on the initial probability space.

DEFINITION 2.1. The Markov process $\left\{\mu_{t} ; t \geq 0\right\}$ with respect to $\left\{\mathscr{F}_{t} ; t \geq 0\right\}$ in the space $(\mathfrak{W}, \gamma)$ is called evolutionary if for every initial state $\mu_{0} \in \mathfrak{W}$, there exists the measurable function

$$
f: \Omega \times[0,+\infty) \times \mathfrak{X} \longrightarrow \mathfrak{X}
$$

such that 
(1) for every $t>0$, the restriction of $f$ on $\Omega \times[0, t] \times \mathfrak{X}$ is measurable with respect to the $\sigma$-field generated by the product $\mathscr{F}_{t}, \mathscr{B}$, and the Borel $\sigma$ field in $[0, t]$,

(2) for every $t \geq 0$,

$$
\mu_{t}=\mu_{0} \circ f_{t}^{-1}(\operatorname{modP})
$$

(3) for arbitrary $u_{1}, \ldots, u_{n} \in \mathfrak{X}, n \geq 1,\left\{\left(\mu_{t}, f_{t}\left(u_{1}\right), \ldots, f_{t}\left(u_{n}\right)\right)\right.$; $\left.t \geq 0\right\}$ is a Markov process with respect to the flow $\left\{\mathscr{F}_{t}\right\}$.

It is natural to define the evolutionary measure-valued process by the set of probabilities $\left\{Q\left(\mu, t, u_{1}, \ldots, u_{n}\right)\left(\Gamma_{n}\right) ; \mu \in \mathfrak{W}, t \geq 0, u_{1}, \ldots, u_{n} \in \mathfrak{X}, n \geq 1, \Gamma_{n} \in\right.$ $\left.\mathscr{B}_{n}\right\}$. Here the value $Q\left(\mu, t, u_{1}, \ldots, u_{n}\right)\left(\Gamma_{n}\right)$ is the probability for particles which were initially placed in the points $u_{1}, \ldots, u_{n}$ to get the set $\Gamma_{n}$ for the time $t$ under initial distribution of the mass of all particles $\mu$. The next technical statement will be useful.

LEMMA 2.2. Let $\psi: \Omega \times \mathfrak{X} \rightarrow \mathfrak{X}$ be a measurable function and let $\mu$ be a random element in $2 \mathrm{~h}$. Then the image of $\mu, \mu \circ \psi^{-1}$, under the map $\psi$ is the random element in $2 \mathrm{~N}$.

LEMMA 2.3. In Lemma 2.2, if $\psi$ does not depend on $\mu$, and $\psi_{1}$ is a measurable modification of $\psi$, that is,

$$
\psi(u)=\psi_{1}(u)(\bmod P) \quad \forall u \in \mathfrak{X},
$$

then

$$
\mu \circ \psi^{-1}=\mu \circ \psi_{1}^{-1}(\bmod P)
$$

The proof of these two lemmas is standard and omitted.

Now, let $\left\{R\left(u_{1}, \ldots, u_{n}\right), u_{1}, \ldots, u_{n} \in \mathfrak{X}, n \geq 1\right\}$ be a family of consistent finite-dimensional distributions in $\mathfrak{X}$ indexed by the elements of $\mathfrak{X}$. Suppose that

$$
\iint_{\mathfrak{X}^{2}} \rho\left(v_{1}, v_{2}\right) R\left(u, u_{0}\right)\left(d v_{1}, d v_{2}\right) \rightarrow 0, \quad u \longrightarrow u_{0},
$$

for every $u_{0} \in \mathfrak{X}$. Then one can build from $R$ the stochastic kernel on 2b. Consider $\varphi$ which is an $\mathfrak{X}$-valued random process on $\mathfrak{X}$ correspondent to the family $\{R\}$. It follows from (2.7) that $\varphi$ has a measurable modification. For arbitrary measure $\mu \in 2 \mathfrak{W}$, its image $\mu \circ \varphi^{-1}$ is a random element in $2 \mathfrak{W}$ which does not depend on the choice of measurable modification for $\varphi$. Define

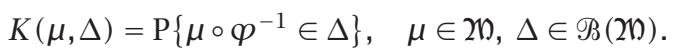

Here $\mathscr{B}(2 \mathrm{D})$ is the Borel $\sigma$-field in $2 \mathrm{n}$. It can be checked that $K$ is the stochastic kernel on $2 \mathfrak{b}$. Note that, in general, arbitrary stochastic kernel on $2 \mathfrak{W}$ cannot be 
obtained in the previous way. Now, suppose that the family $\{R\}$ depends on the measure $\mu$ from 2 n.

LEMmA 2.4. Let the family of the finite-dimensional distributions $\left\{Q\left(\mu, u_{1}\right.\right.$, $\left.\left.\ldots, u_{n}\right) ; \mu \in 2 b, u_{1}, \ldots, u_{n} \in \mathfrak{X}, n \geq 1\right\}$ satisfy the following conditions:

(1) for every fixed $\mu \in 2 \mathfrak{W},\{Q(\mu, \ldots)\}$ is the family of the consistent finitedimensional distributions,

(2) for all $\mu, u \in \mathfrak{X}$,

$$
\lim _{\varepsilon \rightarrow 0+} \sup _{\gamma(\mu, v)<\varepsilon} \sup _{\rho(u, v)<\varepsilon} \iint_{\mathfrak{X}^{2}} \rho\left(p_{1}, p_{2}\right) Q(\nu, u, v)\left(d p_{1}, d p_{2}\right)=0,
$$

(3) there exists $C>0$ for all $u_{1}, \ldots, u_{m} \in \mathfrak{X}$ and all $\mu_{1}, \mu_{2} \in 2 \mathfrak{W}$ such that

$$
\gamma_{m}\left(Q\left(\mu, u_{1}, \ldots, u_{m}\right), Q\left(\mu, u_{1}, \ldots, u_{m}\right)\right) \leq C \gamma\left(\mu_{1}, \mu_{2}\right) .
$$

Then the function

$$
K_{m}\left(\mu, u_{1}, \ldots, u_{m}\right)(\Delta), \quad \mu \in \mathfrak{W}, u_{1}, \ldots, u_{m} \in \mathfrak{X}, \Delta \in \mathscr{B}\left(2 \mathfrak{W} \times \mathfrak{X}^{m}\right),
$$

which is defined by the equality

$$
K_{m}\left(\mu, u_{1}, \ldots, u_{m}\right)(\Delta)=P\left\{\left(\mu \circ \varphi_{\mu}^{-1}, \varphi_{\mu}\left(u_{1}\right), \ldots, \varphi_{\mu}\left(u_{m}\right)\right) \in \Delta\right\},
$$

is the stochastic kernel on $2 \mathfrak{W} \times \mathfrak{X}^{m}$ and the measure $K_{m}\left(\mu, u_{1}, \ldots, u_{m}\right)$ continuously depends on $\mu$ in the Wasserstein metric. Here $\varphi_{\mu}$ is the random map which is built from the family $\{Q(\mu, \ldots)\}$ as in Lemma 2.3.

Proof. It is sufficient to prove only the mentioned continuity. Let $f: 2 \mathfrak{b} \times$ $\mathfrak{X}^{m} \rightarrow \mathbb{R}$ be bounded and satisfy the Lipschitz condition. For the sequence $\left\{\left(\mu_{N}\right.\right.$, $\left.\left.u_{1}^{N}, \ldots, u_{m}^{N}\right)\right\}$ which is convergent to $\left(\mu, u_{1}, \ldots, u_{m}\right)$ as $N \rightarrow \infty$, consider the difference

$\int_{2 n \times \mathfrak{X}^{m}} f\left(v, q_{1}, \ldots, q_{m}\right)\left(K_{m}\left(\mu_{N}, u_{1}^{N}, \ldots, u_{m}^{N}\right)-K_{m}\left(\mu, u_{1}, \ldots, u_{m}\right)\right) d v d q_{1} \cdots d q_{m}$.

We build the random maps $\varphi_{N}$ and $\varphi$ correspondent to the measures $\mu_{N}$ and $\mu$ on the joint probability space. Then (2.13) can be rewritten as follows:

$$
\begin{aligned}
& \left|E f\left(\mu_{N} \circ \varphi_{N}^{-1}, \varphi_{N}\left(u_{1}^{N}\right), \ldots, \varphi_{N}\left(u_{m}^{N}\right)\right)-f\left(\mu \circ \varphi^{-1}, \varphi\left(u_{1}\right), \ldots, \varphi\left(u_{m}\right)\right)\right| \\
& \quad \leq \operatorname{CE\gamma }\left(\mu_{N} \circ \varphi_{N}^{-1}, \mu \circ \varphi^{-1}\right)+C E \sum_{k=1}^{m} \rho\left(\varphi_{N}\left(u_{k}^{N}\right), \varphi\left(u_{k}\right)\right) .
\end{aligned}
$$

Consider every summand separately.

Now,

$$
\begin{aligned}
E \gamma\left(\mu_{N} \circ \varphi_{N}^{-1}, \mu \circ \varphi^{-1}\right) \\
\quad \leq E \gamma\left(\mu_{N} \circ \varphi_{N}^{-1}, \mu_{N} \circ \varphi^{-1}\right)+E \gamma\left(\mu_{N} \circ \varphi^{-1}, \mu \circ \varphi^{-1}\right) .
\end{aligned}
$$


We prove that for every bounded Lipschitz function $h: \mathfrak{X} \rightarrow \mathbb{R}$,

$$
\lim _{N \rightarrow \infty} E\left|\int_{\mathfrak{X}}\left[h\left(\varphi_{N}(u)\right)-h(\varphi(u))\right] \mu_{N}(d u)\right|=0 .
$$

In fact,

$$
\begin{aligned}
E \mid \int_{\mathfrak{X}}\left[h\left(\varphi_{N}(u)\right)\right. & -h(\varphi(u))] \mu_{N}(d u) \mid \\
& \leq C E \int_{\mathscr{X}} \rho\left(\varphi_{N}(u), \varphi(u)\right) \mu_{N}(d u) \\
& =\lim _{n \rightarrow \infty} C E \sum_{k=1}^{n} \rho\left(\varphi_{N}\left(u_{k}^{n}\right), \varphi\left(u_{k}^{n}\right)\right) a_{k}^{n} .
\end{aligned}
$$

Here the discrete measures $\sum_{k=1}^{n} a_{k}^{n} \delta_{u_{k}^{n}}$ weakly converge to $\mu_{N}$ as $n \rightarrow \infty$. Now build the joint probability space for $\varphi_{N}$ and $\varphi$ in a special way. Consider, for fixed $n \geq 1$, the space $\mathfrak{X}^{\mathfrak{X}} \times \mathfrak{X}^{\mathfrak{X}}$ with the $\sigma$-field, which is generated by the cylindrical sets.

Define the probability measure by its finite-dimensional distributions. For fixed $\varepsilon>0$, consider on $\mathfrak{X}^{2 n}=\mathfrak{X}^{n} \times \mathfrak{X}^{n}$ the measure $\varkappa \in C\left(Q\left(\mu_{N}, u_{1}^{n}, \ldots, u_{n}^{n}\right)\right.$, $\left.Q\left(\mu, u_{1}^{n}, \ldots, u_{n}^{n}\right)\right)$ for which

$$
\begin{aligned}
& \int_{\mathfrak{X}^{2 n}} \rho_{n}(p, q) \varkappa\left(d p_{1}, \ldots, d p_{n}, d q_{1}, \ldots, d q_{n}\right) \\
& \quad \leq \gamma_{n}\left(Q\left(\mu_{N}, u_{1}^{n}, \ldots, u_{n}^{n}\right), Q\left(\mu, u_{1}^{n}, \ldots, u_{n}^{n}\right)\right)+\varepsilon .
\end{aligned}
$$

For the arbitrary sets $u_{1}^{n}, \ldots, u_{n}^{n}, v_{1}, \ldots, v_{m}, r_{1}, \ldots, r_{l} \in \mathcal{X}$, define the correspondent measure on $\mathfrak{X}^{2 n+m+l}$ as follows. For $p_{1}, \ldots, p_{n} \in \mathfrak{X}$, let $S_{N}\left(p_{1}, \ldots, p_{n}\right)$ and $S\left(p_{1}, \ldots, p_{n}\right)$ be conditional measures correspondent to the measures $Q\left(\mu_{N}\right.$, $\left.u_{1}^{n}, \ldots, u_{n}^{n}, v_{1}, \ldots, v_{m}\right)$ and $Q\left(\mu_{N}, u_{1}^{n}, \ldots, u_{n}^{n}, r_{1}, \ldots, r_{l}\right)$. Define the measure

$$
\begin{aligned}
& U\left(u_{1}^{n}, \ldots, u_{n}^{n}, v_{1}, \ldots, v_{m}, u_{1}^{n}, \ldots, u_{n}^{n}, r_{1}, \ldots, r_{l}\right)\left(\Delta_{2 n} \times \Delta_{m} \times \Delta_{l}\right) \\
& \quad=\int_{\Delta_{2 n}} S_{n}\left(p_{1}, \ldots, p_{n}\right)\left(\Delta_{m}\right) S\left(q_{1}, \ldots, q_{n}\right)\left(\Delta_{l}\right) \varkappa\left(d p_{1}, \ldots, d p_{n}, d q_{1}, \ldots, d q_{n}\right) .
\end{aligned}
$$

Here $\Delta_{2 n}, \Delta_{m}$, and $\Delta_{l}$ are the Borel subsets of $\mathfrak{X}^{2 n}, \mathfrak{X}^{m}$, and $\mathfrak{X}^{l}$. The family $\{U\}$ is the consistent family of the finite-dimensional distributions. Correspondent random maps $\varphi_{N}$ and $\varphi$ have the desired distributions. Now

$$
\begin{aligned}
E \sum_{k=1}^{n} \rho\left(\varphi_{N}\left(u_{k}^{n}\right), \varphi\left(u_{k}^{n}\right)\right) a_{k} & \leq E \max _{k=1, \ldots, n} \rho\left(\varphi_{N}\left(u_{k}^{n}\right), \varphi\left(u_{k}^{n}\right)\right) \\
& <\gamma_{m}\left(Q\left(\mu_{N}, u_{1}^{n}, \ldots, u_{n}^{n}\right), Q\left(\mu, u_{1}^{n}, \ldots, u_{n}^{n}\right)\right)+\varepsilon \\
& \leq C_{1} \gamma\left(\mu_{N}, \mu\right)+\varepsilon .
\end{aligned}
$$


So,

$$
E \gamma\left(\mu_{N} \circ \varphi_{N}^{-1}, \mu_{N} \circ \varphi^{-1}\right) \longrightarrow 0, \quad N \longrightarrow \infty .
$$

Now consider $E \gamma\left(\mu_{N} \circ \varphi^{-1}, \mu \circ \varphi^{-1}\right)$. For the same function $h$ as before, consider

$$
E\left|\int_{\mathfrak{X}} h(\varphi(u)) \mu_{N}(d u)-\int_{\mathfrak{X}} h(\varphi(u)) \mu(d u)\right| .
$$

Since $\left\{\mu_{N} ; N \geq 1\right\}$ converges weakly to $\mu$ as $N \rightarrow \infty$, there exist such a probability space $\left(\Omega_{1}, \mathscr{F}_{1}, \mathrm{P}_{1}\right)$ and a sequence of $\mathfrak{X}$-valued random elements $\left\{\xi_{N} ; N \geq 1\right\}$ on it, that is,

$$
\begin{gathered}
\xi_{N} \longrightarrow \xi_{0}, \quad N \longrightarrow \infty\left(\bmod P_{1}\right), \\
{[2 p t] \mathrm{P}_{1} \circ \xi_{N}^{-1}=\mu_{N}, \quad N \geq 1, \mathrm{P}_{1} \circ \xi_{0}^{-1}=\mu .}
\end{gathered}
$$

Then

$$
\begin{aligned}
E \mid \int_{\mathfrak{X}} h(\varphi(u)) \mu_{N}(d u) & -\int_{\mathscr{X}} h(\varphi(u)) \mu(d u) \mid \\
& \leq E E_{1}\left|h\left(\varphi\left(\xi_{N}\right)\right)-h\left(\varphi\left(\xi_{0}\right)\right)\right| \\
& \leq C E_{1} E \rho\left(\varphi\left(\xi_{N}\right), \varphi\left(\xi_{0}\right)\right) .
\end{aligned}
$$

Due to condition (2) of the lemma,

$$
E \rho(\varphi(u), \varphi(v)) \longrightarrow 0, \quad u \longrightarrow v
$$

So,

$$
E_{1} E \rho\left(\varphi\left(\xi_{N}\right), \varphi\left(\xi_{0}\right)\right) \longrightarrow 0, \quad N \rightarrow \infty
$$

To complete the proof, it remains to consider

$$
E \sum_{k=1}^{m} \rho\left(\varphi_{N}\left(u_{k}^{N}\right), \varphi\left(u_{k}\right)\right) .
$$

Now

$$
E \rho\left(\varphi_{N}\left(u_{k}^{N}\right), \varphi\left(u_{k}\right)\right) \leq E \rho\left(\varphi_{N}\left(u_{k}^{N}\right), \varphi_{N}\left(u_{k}\right)\right)+E \rho\left(\varphi_{N}\left(u_{k}\right), \varphi\left(u_{k}\right)\right) .
$$

The first summand tends to zero due to condition (1), and the convergence of the second is already checked. The lemma is proved.

COROLLARY 2.5. Under the conditions of Lemma 2.4, the function

$$
K(\mu, \Delta)=P\left\{\mu \circ \varphi_{\mu}^{-1} \in \Delta\right\}, \quad \mu \in \mathfrak{W}, \Delta \in \mathscr{B}(\mathfrak{W}),
$$

is the stochastic kernel. 
Using Lemma 2.4, it is possible to construct the evolutionary Markov processes using probabilities which describe the motion of the separate particles. The following theorem holds.

THEOREM 2.6. Let the family $\left\{Q\left(t, \mu, u_{1}, \ldots, u_{n}\right) ; t \geq 0, \mu \in \mathfrak{W}, u_{1}, \ldots, u_{n} \in \mathfrak{X}\right\}$ satisfy the conditions of Lemma 2.4 under fixed $t \geq 0$, and for all $t, s \geq 0$, all $u_{1}, \ldots, u_{n}, v_{1}, \ldots, v_{m} \in \mathfrak{X}$, all $\Delta \in \mathscr{B}$, and all $\Gamma \in \mathscr{B}\left(\mathfrak{X}^{m}\right)$,

$$
\begin{array}{rl}
\int_{\mathfrak{X}^{n+m}} & Q\left(t+s, \frac{1}{n} \sum_{k=1}^{n} \delta_{u_{k}}, u_{1}, \ldots, u_{n}, v_{1}, \ldots, v_{m}\right)\left(d x_{1}, \ldots, d x_{n}, d y_{1}, \ldots, d y_{m}\right) \\
& \times I_{\Delta}\left(\frac{1}{n} \sum_{k=1}^{n} \delta_{x_{k}}\right) I_{\Gamma}\left(y_{1}, \ldots, y_{m}\right) \\
= & \int_{\mathfrak{X}^{n+m}} Q\left(t, \frac{1}{n} \sum_{k=1}^{n} \delta_{u_{k}}, u_{1}, \ldots, u_{n}, v_{1}, \ldots, v_{m}\right)\left(d x_{1}^{\prime}, \ldots, d x_{n}^{\prime}, d y_{1}^{\prime}, \ldots, d y_{m}^{\prime}\right) \\
\quad \times \int_{\mathfrak{X}^{n+m}} Q\left(s, \frac{1}{n} \sum_{k=1}^{n} \delta_{x_{k}^{\prime}}, x_{1}^{\prime}, \ldots, x_{n}^{\prime}, y_{1}^{\prime}, \ldots, y_{m}^{\prime}\right)\left(d x_{1}^{\prime \prime}, \ldots, d x_{n}^{\prime \prime}, d y_{1}^{\prime \prime}, \ldots, d y_{m}^{\prime \prime}\right) \\
\quad \times I_{\Delta}\left(\frac{1}{n} \sum_{k=1}^{n} \delta_{x_{k}^{\prime \prime}}\right) I_{\Gamma}\left(y_{1}^{\prime \prime}, \ldots, y_{m}^{\prime \prime}\right) .
\end{array}
$$

Then the Markov process constructed with the help of the stochastic kernel

$$
K(t, \mu, \Delta)=P\left\{\mu \circ \varphi_{t, \mu}^{-1} \in \Delta\right\}, \quad t \geq 0, \mu \in \mathfrak{W}, \Delta \in \mathscr{B}(\mathfrak{W}),
$$

is evolutionary. Here $\varphi_{t, \mu}$ is the random map correspondent to the family $\{Q(t$, $\mu, \ldots)\}$.

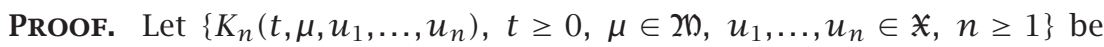
the stochastic kernels which were built in Lemma 2.4. Consider the following consistent family of the finite-dimensional distributions on $[0,+\infty) \times \mathfrak{X}$. For $0 \leq t_{0}<t_{1}<\cdots<t_{m}, u_{1}, \ldots, u_{n} \in \mathfrak{X}$, define the probability measure in $(2 \mathfrak{W} \times \mathfrak{X})^{(m+1) n}$ correspondent to the set of parameters $\left\{\left(t_{i}, u_{j}\right) ; i=0, \ldots, m, j=\right.$ $1, \ldots, n\}$ as follows:

$$
\begin{aligned}
& R\left(t_{0}, \ldots, t_{m}, u_{1}, \ldots, u_{n}\right)\left(\prod_{k=0}^{m} \prod_{j=1}^{n} \Delta_{j}^{k} \times \Gamma_{j}^{k}\right) \\
& =\prod_{k=1}^{n}\left(I_{\Delta_{k}^{0}}(\mu) I_{\Gamma_{k}^{0}}\left(u_{k}\right)\right) \\
& \quad \times \int_{2 \emptyset \times \mathfrak{X}^{n}} K_{n}\left(\mu, u_{1}, \ldots, u_{n}\right)\left(d v_{1}, d v_{1}^{1}, \ldots, d v_{n}^{1}\right) \prod_{k=1}^{n}\left(I_{\Delta_{k}^{1}}\left(v_{1}\right) I_{\Gamma_{k}^{1}}\left(v_{k}^{1}\right)\right)
\end{aligned}
$$




$$
\begin{aligned}
& \times \int_{2 \emptyset \times \mathfrak{*}^{n}} K_{n}\left(v_{1}, v_{1}^{1}, \ldots, v_{n}^{1}\right)\left(d v_{2}, d v_{1}^{2}, \ldots, d v_{n}^{2}\right) \prod_{k=1}^{n}\left(I_{\Delta_{k}^{2}}\left(v_{2}\right) I_{\Gamma_{k}^{2}}\left(v_{k}^{2}\right)\right) \ldots \\
& \times \int_{2 m \times *^{n}} K_{n}\left(v_{m-1}, v_{1}^{m-1}, \ldots, v_{n}^{m-1}\right)\left(d v_{m}, d v_{1}^{m}, \ldots, d v_{n}^{m}\right) \\
& \times \prod_{k=1}^{n}\left(I_{\Delta_{k}^{m}}\left(v_{m}\right) I_{\Gamma_{k}^{m}}\left(v_{k}^{m}\right)\right) \text {. }
\end{aligned}
$$

Due to Lemma 2.4 and the condition of the theorem, $\{R\}$ is the consistent family of the finite-dimensional distributions. Consider the random process $\{(\mu(t, u), \varphi(t, u)) ; t \geq 0, u \in \mathfrak{X}\}$ correspondent to $\{R\}$. Note that due to the construction of $\{R\}, \mu$ can be chosen in a way independent from $u$. Moreover, under fixed $t \geq 0, \varphi$ is stochastically continuous with respect to $u$. It can easily be checked that the random process $\left\{\left(\mu(t), \varphi\left(t, u_{1}\right), \ldots, \varphi\left(t, u_{n}\right)\right) ; t \geq 0\right\}$ is Markov. For every $t \geq 0$,

$$
E \gamma\left(\mu(t), \mu(0) \circ \varphi(t, \cdot)^{-1}\right)=0 .
$$

The last equality follows from the approximation by the discrete measures. Thus the theorem is proved.

3. Equations driven by the measures. In this section, we consider the stochastic equation from Example 1.1. Our measure-valued process will be constructed with the help of the following stochastic differential equation:

$$
\begin{gathered}
d x(u, t)=a\left(x(u, t), \mu_{t}\right) d t+b\left(x(u, t), \mu_{t}\right) d w(t), \\
x(u, 0)=u, \quad u \in \mathbb{R}^{d}, \\
\mu_{t}=\mu_{0} \cdot x(\cdot, t)^{-1} .
\end{gathered}
$$

Here, coefficients $a$ and $b$ are the jointly Lipschitz functions $a: \mathbb{R}^{d} \times 2 \mathfrak{V}_{2} \rightarrow$

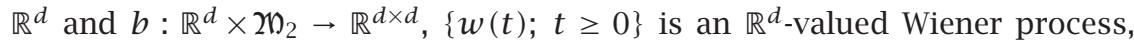
$\mu_{t}=\mu_{0} \cdot x(\cdot, t)^{-1}$ is an image of the initial measure $\mu_{0}$ under the random map $x(\cdot, t)$. It has been proved in [1] that under our conditions on the coefficients, (3.1) has a unique solution. The process $\left\{\mu_{t} ; t \geq 0\right\}$ has a strongly Markovian property.

Here, we prove the existence of the generalized local time for the process $\left\{\mu_{t} ; t \geq 0\right\}$. We consider the functional of the form

$$
L(u, t)=\int_{0}^{t} \int_{\mathbb{R}^{d}} \delta_{u}(x(v, s)) \mu_{0}(d v) d s
$$

in the case when the measure $\mu_{0}$ is concentrated on the $(d-1)$-dimensional surface $S_{0}$ and is absolutely continuous with respect to surface measure. This 
formal expression is a some kind of the generalization of the notion of local time for the measure-valued processes. There are two important reasons which lead to the existence of the local time. First, the trajectories of the single particles, from which our process is composed, can have the usual local time at the point $u$. Second, our process consists of the smooth measures. We will consider the special type of the measure-valued processes, which is organized from the mass of the interacting particles. But in the case $d \geq 2$, one cannot expect the existence of the local time for the trajectory of the single particle. Also we will use the singular initial mass distribution.

Consider the random flow $\{x(\cdot, t) ; t \in[0,1]\}$. In order to get the necessary properties of $x$, we treat (3.1) as the usual stochastic differential equation for $x$ with the random coefficients

$$
\tilde{a}(x, t)=a\left(x, \mu_{t}\right), \quad \tilde{b}(x, t)=b\left(x, \mu_{t}\right),
$$

where $x \in \mathbb{R}^{d}, t \in[0,1]$.

Then, under appropriate conditions on $a$ and $b$, we can derive the same properties of $x(\cdot, t), t \geq 0$, as the properties of the stochastic flow in the sense of Kunita. The next lemma is a straightforward consequence of [5, Theorem 4.4.2, page 148].

LEMMA 3.1. Let the coefficients $a$ and $b$ of (2.13) satisfy the following conditions:

(1) for all $k=0,1,2,3$,

$$
\frac{\partial^{k} a}{\partial u^{k}}, \frac{\partial^{k} b}{\partial u^{k}} \in C\left(\mathbb{R}^{d} \times 2 \mathfrak{W}_{2}\right)
$$

(2) for all $k=0,1,2,3$,

$$
\sup _{\mathbb{R}^{d} \times 2 b_{2}}\left(\left\|\frac{\partial^{k} a}{\partial u^{k}}\right\|+\left\|\frac{\partial^{k} b}{\partial u^{k}}\right\|\right)<+\infty .
$$

Then the random field $x(u, t), u \in \mathbb{R}^{d}, t \in[0,1]$, has a modification such that

(1) $x \in C\left(\mathbb{R}^{d} \times[0,1]\right)$ a.s.,

(2) $x(\cdot, t)$ is a $C^{2}$-diffeomorphism $\mathbb{R}^{d}$ onto $\mathbb{R}^{d}$ for every $t \in[0,1]$ a.s.,

(3) the inverse mappings $x(\cdot, t)^{-1}, t \in[0,1]$, are such that, for every $u \in$ $\mathbb{R}^{d},\left\{x(u, t)^{-1}, t \in[0,1]\right\}$ is the Itô process with the differential

$$
d x(u, t)^{-1}=\alpha(u, t) d t+\beta(u, t) d w(t)
$$

where $\alpha$ and $\beta$ have a continuous modification with respect to $u$ and $t$.

Now we consider the surface in $\mathbb{R}^{d}$

$$
S_{0}=\left\{u=\left(u^{1}, \ldots, u^{d}\right): u^{d}=0\right\} .
$$


Denote by $S_{t}$ the image of $S_{0}$ under the random map $x(\cdot, t)$. Due to the properties of $x(\cdot, t)$, which were mentioned in Lemma 3.1, $S_{t}$ is a smooth surface of codimension one. Our aim is to register the cases when the fixed point (zero in the sequel without loss of generality) belongs to $S_{t}$; that is, we will consider the functional

$$
\zeta(t)=\int_{0}^{t} \delta_{0}\left(x(0, s)_{d}^{-1}\right) d s
$$

where $\delta_{0}$ is a $\delta$-function on $\mathbb{R}$ concentrated at the original and $x(0, s)_{d}^{-1}$ is the last coordinate of $x(0, s)^{-1}$. In order to define $\zeta$ in a proper way, we use the Tanaka approach (see [4]). Consider the function $h \in C(\mathbb{R})$ which is even, nonnegative, finite, and has the property

$$
\int_{\mathbb{R}} h(r) d r=1
$$

Let

$$
h_{n}(r)=n h(r n), \quad r \in \mathbb{R}, n \geq 1
$$

Now the following lemma holds.

LEMMA 3.2. In addition to the conditions of Lemma 3.1, let b be uniformly nondegenerate with respect to $u$ and $t$. Then there exists the limit

$$
\zeta(t)=P-\lim _{n \rightarrow \infty} \int_{0}^{t} h_{n}\left(x(0, s)_{d}^{-1}\right) d s
$$

where $\{\zeta(t) ; t \in[0,1]\}$ has a modification which is the nondecreasing right continuous process.

Proof. It follows from Lemma 3.1 that

$$
x(0, s)_{d}^{-1}=\int_{0}^{s} \alpha(0, r)_{d} d r+\sum_{j=1}^{d} \int_{0}^{s} \beta(0, r)_{d j} d w_{j}(r),
$$

where

$$
\beta(0, r)=\left(\beta(0, r)_{i j}\right)_{i j=1}^{d}, \quad w(r)=\left(w_{1}(r), \ldots, w_{d}(r)\right)
$$

Now, using the Itô formula in the process $x(0, s)_{d}^{-1}$ and the function

$$
F_{n}(x)=\int_{-\infty}^{x} h_{n}(z) d z d y
$$


we get

$$
\begin{aligned}
& F_{n}\left(x(0, t)_{d}^{-1}\right)-F_{n}(0) \\
& \qquad \int_{0}^{t} F_{n}^{\prime}\left(x(0, s)_{d}^{-1}\right) \alpha(0, s)_{d} d s+\frac{1}{2} \int_{0}^{t} h_{n}\left(x(0, s)_{d}^{-1}\right) \sum_{j=1}^{d}\left(\beta(0, s)_{d j}\right)^{2} d s \\
& \quad+\int_{0}^{t} F_{n}^{\prime}\left(x(0, s)_{d}^{-1}\right) \sum_{j=1}^{d} \beta(0, s)_{d j} d w_{j}(s) .
\end{aligned}
$$

By the usual limit arguments, it follows that there exists a limit

$$
L_{2}-\lim _{n \rightarrow \infty} \int_{0}^{t} h_{n}\left(x(0, s)_{d}^{-1}\right) \sum_{j=1}^{d}\left(\beta(0, s)_{d j}\right)^{2} d s .
$$

Note that it follows from the explicit representation of the stochastic differential of $x(u, t)^{-1}$ in [5] that, under the condition of nondegeneracy of $b$, the sum

$$
\sum_{j=1}^{d}\left(\beta(0, s)_{d j}\right)^{2}
$$

satisfies the relation

$$
\min _{[0,1]} \sum_{j=1}^{d}\left(\beta(0, s)_{d j}\right)^{2}>0 \quad \text { a.s. }
$$

We denote

$$
\tilde{\beta}(s)=\sum_{j=1}^{d}\left(\beta(0, s)_{d j}\right)^{2}, \quad s \in[0, t] .
$$

Now consider the arbitrary subsequence of $\left\{\int_{0}^{t} h_{n}\left(x(0, s)_{d}^{-1}\right) d s ; n \geq 1\right\}$. Using the diagonal method, we can choose from this subsequence the subsubsequence which we will shortly denote by $\left\{\int_{0}^{t} h_{n_{k}}\left(x(0, s)_{d}^{-1}\right) d s ; k \geq 1\right\}$ such that it satisfies following relations. There exists the set $\Omega_{0} \subset \Omega$ of probability one such that, for every $\omega \in \Omega_{0}$,

(1) $\tilde{\beta}(\omega) \in C([0, t]), \min _{[0, t]} \tilde{\beta}(\omega)>0$,

(2) for all $r_{1}, r_{2} \in \mathbb{Q} \cap[0, t]$, there exists a limit

$$
\lim _{k \rightarrow \infty} \int_{r_{1}}^{r_{2}} h_{n_{k}}\left(x(0 ; s)_{d}^{-1}\right) \tilde{\beta}(s) d s,
$$

(3) $\sup _{k \geq 1} \int_{0}^{t} h_{n_{k}}\left(x(0, s)_{d}^{-1}\right) d s<+\infty$. 
It follows from (1), (2), and (3) that for every $\omega \in \Omega_{0}$ and every $\varphi \in C([0, t])$, there exists a limit

$$
\lim _{k \rightarrow \infty} \int_{0}^{t} h_{n_{k}}\left(x(0, s)_{d}^{-1}\right) \varphi(s) d s
$$

In fact, for fixed $\omega$ using uniform continuity of the functions $\varphi$ and $\tilde{\beta}(\omega)$, one can construct the sequence of the functions $\left\{\varphi_{m} ; m \geq 1\right\}$ such that

(4) for every $m \geq 1$, there exists the partition $0=t_{0}^{m}<\cdots<t_{N_{m}}^{m}=t$ of the rational numbers (excluding $t$ ) for which

$$
\varphi_{m}(s)=\frac{\varphi\left(t_{j}^{m}\right)}{\tilde{\beta}\left(t_{j}^{m}\right)} \tilde{\beta}(s), \quad \varphi_{m}(t)=\frac{\varphi\left(t_{N_{m}-1}^{m}\right)}{\tilde{\beta}\left(t_{N_{m}-1}^{m}\right)} \tilde{\beta}(s),
$$

for all $j=0, \ldots, N_{m}-1$ and all $s \in\left(t_{j}^{m} ; t_{j+1}^{m}\right)$,

(5) $\sup _{[0, t]}\left|\varphi_{m}-\varphi\right| \rightarrow 0, m \rightarrow \infty$,

(6) for every $m \geq 1$, there exists the limit

$$
\lim _{k \rightarrow \infty} \int_{0}^{t} h_{n_{k}}\left(x(0, s)_{d}^{-1}\right) \varphi_{m}(s) d s .
$$

Now, using (5) and (3), we conclude that there exists the limit (3.21). So, substituting $\varphi \equiv 1$, we prove the statement of the lemma.

THEOREM 3.3. Suppose that the coefficients of (2.13) satisfy the conditions of Lemma 3.2. Let the initial measure $\mu_{0}$ be concentrated on the surface $S_{0}$ and have a continuous density $p$ with respect to the Lebesgue measure on this surface. Then there exists the limit in probability

$$
P-\lim _{n \rightarrow \infty} \int_{0}^{t} \int_{\mathbb{R}^{d}} f_{n}(u) \mu_{s}(d u) d s,
$$

where $f_{n}(v)=n^{d} \cdot f(n v)$ and $f \in C_{0}^{1}\left(\mathbb{R}^{d}\right)$ is nonnegative, spherically symmetric with $\int_{\mathbb{R}^{d}} f(u) d u=1$.

Proof. We consider the stochastic flow $\{x(\cdot, s), s \in[0, t]\}$, which is correspondent to the initial measure $\mu_{0}$ via (2.13). Then

$$
\int_{0}^{t} \int_{\mathbb{R}^{d}} f_{n}(u) \mu_{s}(d u) d s=\int_{0}^{t} \int_{\mathbb{R}^{d}} f_{n}(x(u, s)) \mu_{0}(d u) d s .
$$

We define the functions

$$
\begin{gathered}
\varphi_{n}(s, u)=\int_{S_{0}} f_{n}\left(x\left(v_{1}, \ldots, v_{d-1}, u, s\right)\right) p\left(v_{1}, \ldots, v_{d-1}, 0\right) d v_{1}, \ldots d v_{d-1}, \\
u \in \mathbb{R}, s \in[0,1] \\
\psi_{n}(s, u)=\varphi_{n}\left(s, u-x(0, s)_{d}^{-1}\right), \quad u \in \mathbb{R}, s \in[0,1]
\end{gathered}
$$


Using the diffeomorphic property of the flow $x$ and the change of variables formula, one can check that there exist a continuous random process $\{J(t) ; t \in$ $[0,1]\}$ and the deterministic sequence of functions $\left\{\boldsymbol{x}_{n} ; n \geq 1\right\}$ on $\mathbb{R}$ such that

(1) for all $n \geq 1$, supp $\varkappa_{n} \subset[-1,1], \varkappa_{n} \geq 0$,

(2) $M \int_{0}^{1}\left[\psi_{n}\left(s, x(0, s)_{d}^{-1}\right)-J(s) \varkappa_{n}\left(x(0, s)_{d}^{-1}\right)\right]^{2} d s \rightarrow 0, n \rightarrow \infty$,

(3) $\left\{\varkappa_{n} ; n \geq 1\right\}$ satisfies the conditions of Lemma 3.2.

Note that

$$
\int_{\mathbb{R}^{d}} f_{n}(v) \mu_{s}(d v)=\psi_{n}\left(s, x(0, s)_{d}^{-1}\right) \quad \text { a.s. }
$$

So, due to Lemma 3.2, for every $t \in[0,1]$, there exists the limit in probability

$$
\text { P- } \lim _{n \rightarrow \infty} \int_{0}^{t} \psi_{n}\left(s, x(0, s)_{d}^{-1}\right) d s=\mathrm{P}-\lim _{n \rightarrow \infty} \int_{0}^{t} J(s) \varkappa_{n}\left(x(0, s)_{d}^{-1}\right) d s .
$$

Thus the theorem is proved.

Note that, in the one-dimensional case, the local time for a process of this type was considered in [2].

Now consider another example of the evolutionary measure-valued process.

EXAMPLE 3.4. Let $(\mathfrak{X}, \rho)$ be a compact metric space and let $\left\{G_{s}^{t} ; 0 \leq s \leq t\right\}$ be a stochastic semigroup of continuous mappings in $\mathfrak{X}[6]$ with the independent homogeneous increments, that is,

(1) $G_{s}^{t} \in C(\mathfrak{X}, \mathfrak{X})$ a.s.,

(2) $G_{s}^{s}$ is an identity map for all $s \geq 0$,

(3) $G_{s}^{t}\left(G_{r}^{s}\right)=G_{r}^{t}$ a.s., $0 \leq r \leq s \leq t$,

(4) $G_{t_{1}}^{t_{2}}, G_{t_{2}}^{t_{3}}, \ldots, G_{t_{n-1}}^{t_{n}}$ are independent for all $t_{1} \leq t_{2} \leq \cdots \leq t_{n}$,

(5) $G_{t_{1}}^{s_{1}}$ and $G_{t_{2}}^{s_{2}}$ are equidistributed under $s_{1}-t_{1}=s_{2}-t_{2} \geq 0$.

Define the Markov process in the space $2 \mathfrak{W} \times C(\mathfrak{X}, \mathfrak{X})$ as follows. For arbitrary $\varkappa \in \mathfrak{W}, g \in C(\mathfrak{X}, \mathfrak{X})$, denote $\varkappa_{t}=\varkappa \circ\left(G_{0}^{t}\right)^{-1}$ and $g_{t}=G_{0}^{t}(g), t \geq 0$. Consider for positive $h \in C(\mathfrak{X})$ the additive functional from $\left\{\left(\varkappa_{t}, g_{t}\right) ; t \geq 0\right\}$ of the form

$$
\varphi_{s}^{t}=\int_{s}^{t} \int_{\mathfrak{X}} h(u) \varkappa_{T}(d u) d \tau, \quad 0 \leq s \leq t
$$

Let $\left\{\psi_{0}^{t} ; t \geq 0\right\}$ be the inverse function for $\left\{\varphi_{0}^{t} ; t \geq 0\right\}$. Consider $\left\{\mu_{t}=\varkappa_{\psi_{0}^{t}} ; t \geq\right.$ $0\}$. This is a Markov evolutionary measure-valued process, and the correspondent stochastic flow is $f_{t}=G_{0}^{\psi_{0}^{t}}, t \geq 0$.

\section{REFERENCES}

[1] D. A. Dawson, Measure-valued Markov processes, École d'Été de Probabilités de Saint-Flour XXI-1991, Lecture Notes in Math., vol. 1541, Springer-Verlag, Berlin, 1993, pp. 1-260.

[2] A. A. Dorogovtsev and N. Yu. Goncharuk, Local times for measure-valued Itô's stochastic processes, preprint 95-142, 1995. 
[3] A. A. Dorogovtsev and P. Kotelenez, Smooth stationary solutions of quasilinear stochastic partial differential equations, preprint 97-145, 1997.

[4] N. Ikeda and Sh. Watanabe, Stochastic Differential Equations and Diffusion Processes, North-Holland Mathematical Library, vol. 24, North-Holland Publishing, Amsterdam, 1981.

[5] H. Kunita, Stochastic Flows and Stochastic Differential Equations, Cambridge Studies in Advanced Mathematics, vol. 24, Cambridge University Press, Cambridge, 1990.

[6] A. V. Skorokhod, Random Linear Operators, Naukova Dumka, Kiev, 1979.

Andrey A. Dorogovtsev: Institute of Mathematics, National Academy of Sciences of Ukraine, 3 Tereshchenkivska, 01601 Kiev, Ukraine

E-mail address: adoro@imath.kiev.ua 


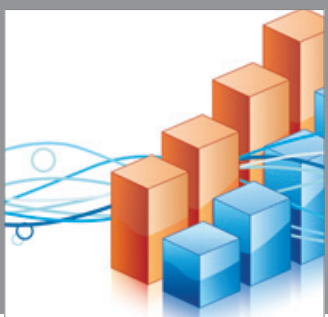

Advances in

Operations Research

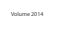

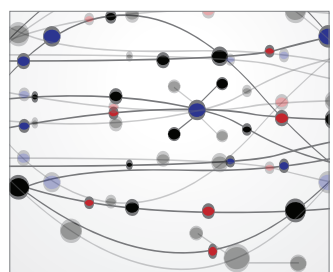

\section{The Scientific} World Journal
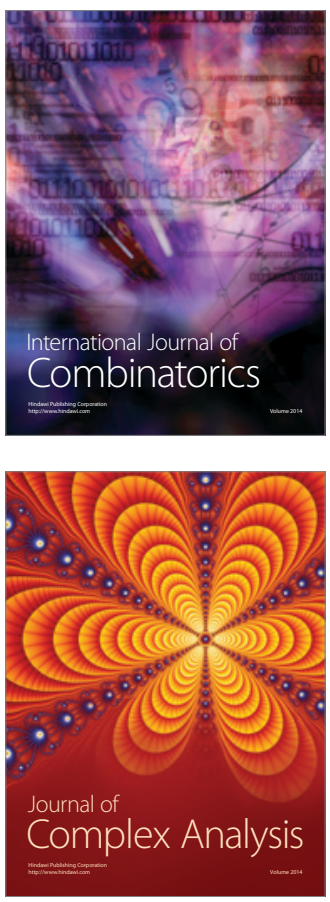

International Journal of

Mathematics and

Mathematical

Sciences
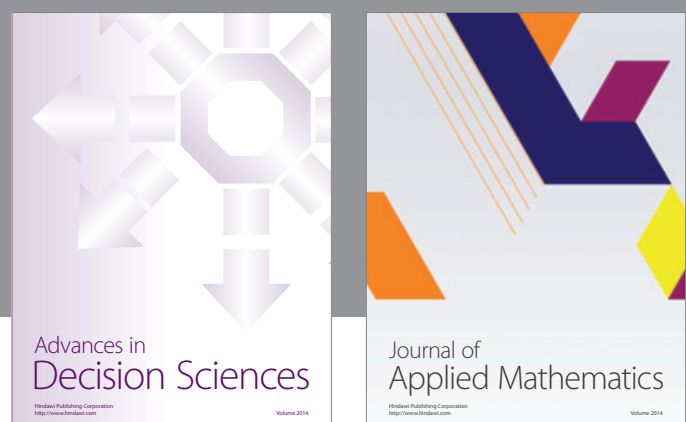

Journal of

Applied Mathematics
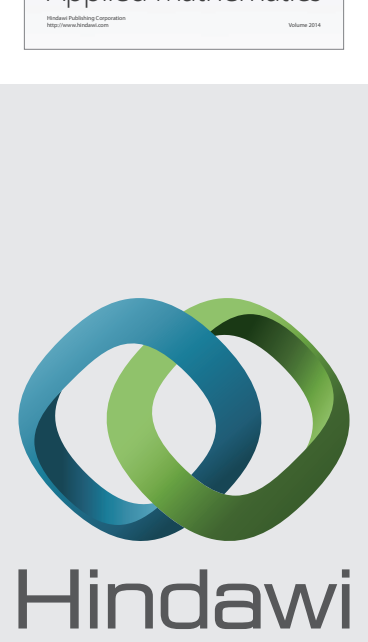

Submit your manuscripts at http://www.hindawi.com
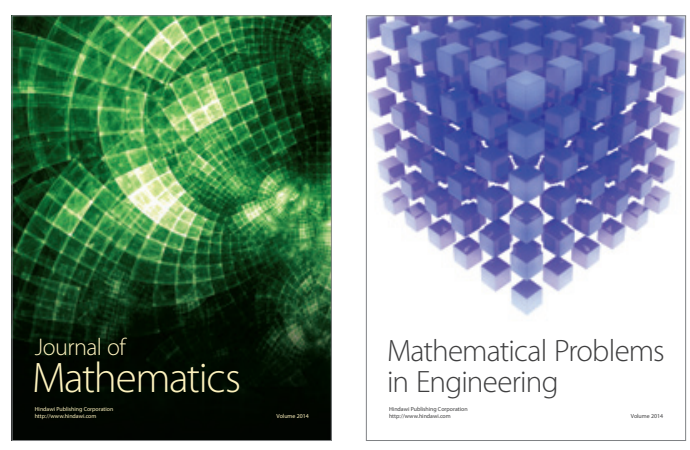

Mathematical Problems in Engineering
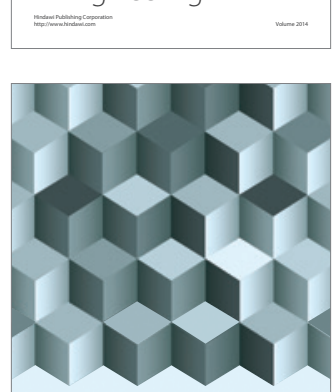

Journal of

Function Spaces
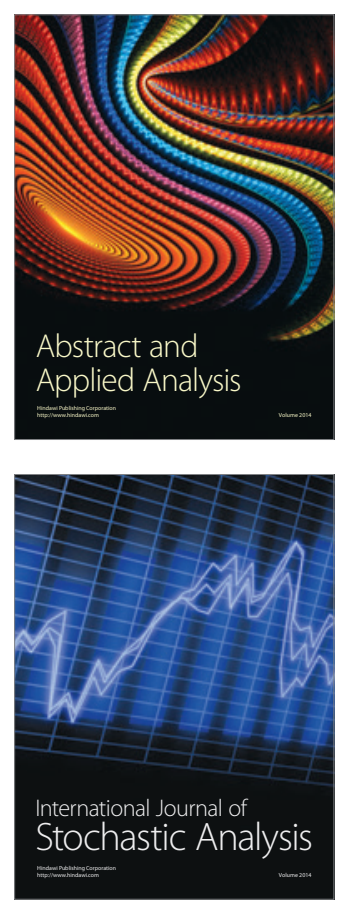

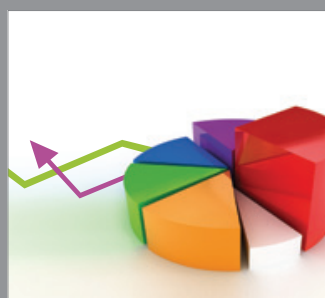

ournal of

Probability and Statistics

Promensencen
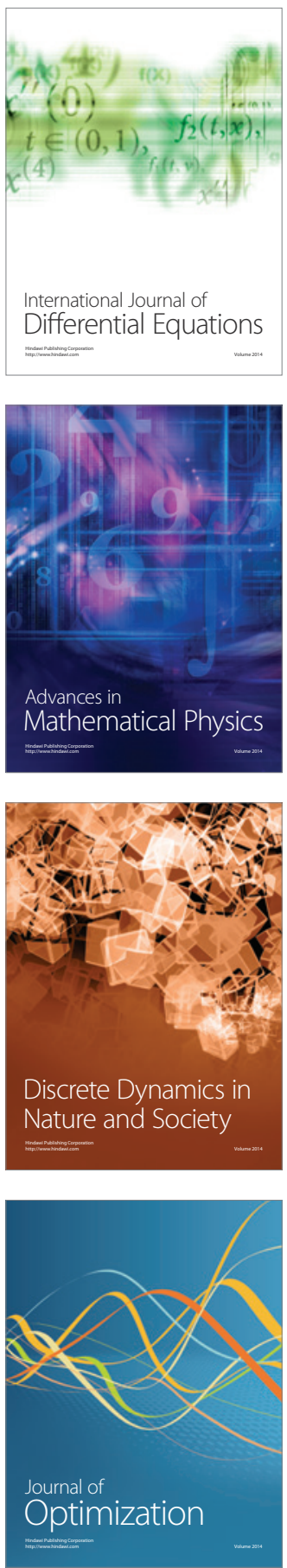